\title{
Stage I Norwood: Optimal technical performance improves outcomes irrespective of preoperative physiologic status or case complexity
}

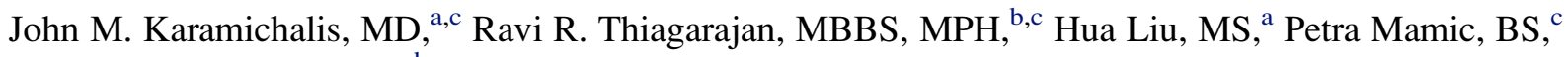 \\ Kimberlee Gauvreau, $\mathrm{ScD},{ }^{\mathrm{b}}$ and Emile A. Bacha, $\mathrm{MD}^{\mathrm{a}, \mathrm{c}}$
}

\begin{abstract}
Objective: Interplay of baseline physiologic status, case complexity, technical performance, and outcomes in high-acuity operations has been poorly defined. This study explored these interactions to determine whether a technically optimal operation can mitigate effects of baseline physiology and high case-complexity on outcomes for the stage I Norwood procedure.
\end{abstract}

\begin{abstract}
Methods: Technical performance was categorized as optimal, adequate, or inadequate from adequacy of the anatomic repair of the stage I subprocedures according to anatomic areas where intervention is performed. Physiological illness severity statuses in preoperative and postoperative periods were determined with Pediatric Risk of Mortality III system, which uses 17 physiologic variables. Case complexity was calculated with Aristotle comprehensive system. All patients undergoing stage I procedure from January 2004 to December 2007 were retrospectively studied.
\end{abstract}

Results: One hundred thirty-five procedures were included. Five were excluded from the technical performance assessment because of inadequate postoperative data. Eighty-one (62.3\%), $26(20 \%)$, and $23(17.7 \%)$, respectively, were scored as optimal, adequate, and inadequate. Overall hospital mortality was $14.1 \%$. Inadequate technical performance, high-complexity Aristotle comprehensive scores, and high preoperative illness severity scores correlated with significantly higher hospital mortality, longer stay, and greater frequency of major postoperative complications. In subgroup analysis of patients with optimal technical performance, outcomes were favorable irrespective of high or low preoperative physiologic illness severity or case complexity.

Conclusions: In stage I Norwood procedures, optimal technical performance attenuated effects of poor preoperative physiologic status and high case complexity, with reduced hospital mortality. Inadequate technical performance resulted in poor outcomes regardless of preoperative status. (J Thorac Cardiovasc Surg 2010;139:962-8)

The stage I Norwood procedure remains one of the most challenging operations in congenital heart surgery. It is technically extremely delicate, and apart from the reduction in pulmonary overcirculation there is no immediate physiologic benefit. We have previously shown that technical performance has a major effect on outcomes, including mortality, of patients undergoing the stage I Norwood operation. ${ }^{1}$ The interplay of baseline physiologic status, anatomic risk factors, technical performance, and postoperative recovery in this patient population has been mainly defined on the basis of patient-specific or anatomic risk factors (prematurity, lower weight, intact atrial septum, smaller ascending aorta, etc) or procedure-specific risk factors (longer

\footnotetext{
From the Departments of Cardiac Surgery a and Cardiology, ${ }^{\mathrm{b}}$ Children's Hospital Boston, and Harvard Medical School, ${ }^{\mathrm{c}}$ Boston, Mass.

Disclosures: None.

Supported by a grant from The Children's Heart Foundation.

Received for publication June 15, 2009; revisions received Sept 8, 2009; accepted for publication Oct 5, 2009; available ahead of print Jan 14, 2010.

Address for reprints: Emile A. Bacha, MD, Department of Cardiac Surgery, Children's Hospital Boston, Harvard Medical School, 300 Longwood Ave, Boston, MA 02115 (E-mail: emile.bacha@cardio.chboston.org).

$0022-5223 / \$ 36.00$

Copyright (c) 2010 by The American Association for Thoracic Surgery doi: $10.1016 /$ j.jtcvs.2009.10.011
}

circulatory arrest time, shunt originating from the neoaorta, management of the ascending aorta, etc). ${ }^{2-5}$

In one risk-stratification system, the Aristotle comprehensive system, ${ }^{6-8}$ preoperative physiologic risk factors were included as part of an assessment of an overall risk profile. The Aristotle score is a complexity score that is based on the patient's baseline physiology and anatomy as well as the type of surgical procedure and its potential for mortality, morbidity, and anticipated technical difficulty. The basic and comprehensive Aristotle score values have been previously described. ${ }^{6-8}$

The Pediatric Risk of Mortality (PRISM), a risk-adjustment tool first published in $1988,{ }^{9}$ is a generic severity-scoring system developed to measure the probability of death on the basis of the hypothesis that preoperative physiologic instability reflects mortality risk. The newer and improved PRISM III, ${ }^{10}$ developed in 1996 , is more accurately calibrated and achieves good discrimination for individual patient mortality risk assessments.

The aim of this study was to define and explore the associations of preoperative baseline physiology, case complexity, technical performance, and postoperative outcomes with these various scoring systems. Our hypothesis was that a technically optimal operation could mitigate the effects 


\section{Abbreviations and Acronyms \\ $\mathrm{CI}=$ confidence interval \\ $\mathrm{ECMO}=$ extracorporeal membrane oxygenation \\ ICU $=$ intensive care unit \\ PRISM $=$ Pediatric Risk of Mortality instrument}

of baseline physiology and high case complexity on outcomes for the stage I Norwood procedure.

\section{MATERIALS AND METHODS}

The Children's Hospital Boston institutional review board approved this study. A waiver of informed consent was obtained. Patient data were rendered anonymous in our database in compliance with the hospital requirements.

\section{Technical Performance Measurement Tool}

The predischarge technical score created in our previous study was used. ${ }^{1}$ In brief, the surgical procedure was divided into individual components defined as subprocedures, which were based on the specific anatomic regions subject to intervention. The subprocedures for the stage I Norwood were as follows: atrial septectomy, aortic root reconstruction (coronary perfusion), proximal aortic (distal to sinotubular junction but proximal to the innominate artery) reconstruction, distal aortic arch reconstruction, and source of pulmonary blood flow. Individual subprocedures were scored as optimal, adequate, or inadequate. The parameters for score assessment were based on clinical, echocardiographic, and cardiac catheterization data. ${ }^{1}$ An overall score for each patient was also defined, wherein the operation was graded as optimal if all subprocedures were optimal, adequate if at least 1 subprocedure was adequate but none were inadequate, and inadequate if 1 or more subprocedures were inadequate. Additional procedures performed, such as valvuloplasties, were taken into account in measuring technical performance, and the scores were modified as necessary by using similar principles. The only modification from the previous score was a downgrading from optimal to adequate if an otherwise optimal technical result was achieved by means of a second cardiopulmonary bypass run.

\section{PRISM III Scoring System}

The PRISM III score has 17 physiologic variables subdivided into 26 ranges, which are age adjusted. These include the worst values of cardiovascular and neurologic vital signs, acid-base balance, blood gas values, chemistry and hematologic tests (systolic blood pressure, heart rate, temperature, pupillary reflexes, acidosis, total carbon dioxide, $\mathrm{pH}$, arterial $\mathrm{Po}_{2}, \mathrm{PCO}_{2}$, glucose, potassium, creatinine, blood urea nitrogen, white blood cell count, prothrombin or partial thromboplastin time, and platelet count) from the first 12 or 24 hours after admission to the intensive care unit (ICU).

PRISM III scores were calculated by using the standardized scoring system $^{10}$ at 24 hours before the stage I Norwood procedure and at 24 and 48 hours separately afterward. Patients with no preoperative arterial blood gas values within 24 hours of the operation, were scored for a $\mathrm{Po}_{2}$ between 42 and $49.9 \mathrm{~mm} \mathrm{Hg}$ ( 3 points), because $\mathrm{Po}_{2}$ falls within this range for most patients undergoing the stage I Norwood procedure.

\section{Aristotle Comprehensive Scoring System}

The basic score value for the stage I Norwood procedure is 14.5. The comprehensive score further adjusts the complexity according to the specific patient and procedural characteristics, including anatomic factors, associated procedures, age, and such other clinical and physiologic factors as shock (persistent or resolved at time of surgery), mechanical ventilation to treat cardiorespiratory failure, and renal, endocrine, neurologic, or hepatic dysfunction. The comprehensive score can add a maximum of 10 additional points to the 14.5 points of the basic score. Five points can be added for the procedure-dependent factors and associated procedures, with another maximum of 5 points for the procedure-independent factors (Table 2). The Aristotle comprehensive score was calculated by using preoperative and perioperative data. $^{8}$

\section{Outcomes}

In-hospital mortality was defined as death before hospital discharge or within 30 days of the operation for discharged patients. Patients transferred back to their referring hospital were followed up through their discharge. A death occurring late at a referring hospital was counted in the mortality figures. Postoperative hospital and intensive care unit stays and ventilation time were measured in days after the stage I operation. Major postoperative complications were defined as stroke or major neurologic deficit, initiation of extracorporeal membrane oxygenation (ECMO), reoperation for bleeding, unplanned reoperation for residual defect, cardiac arrest with cardiopulmonary resuscitation, phrenic nerve paralysis requiring diaphragm plication, and mediastinitis.

\section{Data Collection}

A retrospective review was performed on all patients undergoing stage I Norwood palliation from January 2004 to December 2007 at Children's Hospital Boston. Clinical data (echocardiograms, clinical evaluation, and catheterization results) were collected for assessment. Other variables collected for analysis included surgeon identification and preoperative, procedural, morphologic, and postoperative patient characteristics and outcomes listed in Tables 1, 2, and 3. Patients with inadequate clinical or echocardiographic data relevant to the technical score $(\mathrm{n}=5)$ were excluded from the analysis involving technical performance. Because their PRISM III and Aristotle scores were calculated, however, they were included in other analyses.

\section{Statistical Methods}

Patient and procedural characteristics were summarized with frequencies and percentages for categoric variables and the medians and ranges for continuous variables. Relationships between technical performance score and patient outcomes were assessed with the Pearson $\chi^{2}$ test for categoric variables and the Kruskal-Wallis test for continuous variables. Relationships between PRISM III and Aristotle comprehensive scores and dichotomous patient outcomes were evaluated with the Mann-Whitney test. Associations with continuous outcomes were examined with the Spearman rank correlation coefficient. These analyses were repeated within subgroups defined by technical performance score. The ability of PRISM III and Aristotle scores to discriminate between patients who died in hospital and those who did not was assessed by calculating areas under the receiver operating characteristic curve, and $95 \%$ confidence intervals (CIs) were calculated. Relationships between death and patient and procedural factors were also explored with logistic regression analysis; because of the small number of in-hospital deaths, however, no more than 2 explanatory variables could be included in a single multivariable model. Statistical analysis was performed with SPSS 14.0 for Windows (SPSS Inc, Chicago, Ill).

\section{RESULTS}

One hundred thirty-five patients in the stage I Norwood palliation population were identified. This included all patients who underwent the stage I Norwood procedure, including 3 patients who had attempted biventricular repairs that failed and were subsequently converted to stage I palliation, 1 at a later stage and the other 2 during the same 
TABLE 1. Patient and procedural characteristics $(n=135)$

\begin{tabular}{lc}
\hline Age at operation (d, median and range) & $5(2-42)$ \\
Age $>14 \mathrm{~d}$ at operation (no.) & $7(5.2 \%)$ \\
Weight (kg, median and range) & $3.2(1.3-4.7)$ \\
Weight $<2500 \mathrm{~g}$ (no.) & $21(15.6 \%)$ \\
Prematurity* (no.) & $14(10.4 \%)$ \\
Morphologic variants (no.) & \\
AA/MA & $30(22.2 \%)$ \\
AA/MS & $21(15.6 \%)$ \\
AS/MA & $1(0.7 \%)$ \\
AS/MS & $43(31.9 \%)$ \\
Others & $40(29.6 \%)$ \\
Preoperative catheter-based interventions & $32(23.7 \%)$ \\
$\quad$ (fetal or postnatal) & \\
Fetal interventions & $22(16.3 \%)$ \\
Postnatal & $21(15.6 \%)$ \\
Bypass time (min, median and range) & $145(87-312)$ \\
Crossclamp time (min, median and range) & $63(0-189)$ \\
\hline$A A$, Aortic atresia; $M A$, mitral atresia; $M S$, mitral stenosis; $A S$, aortic stenosis. $*$ Prema- \\
turity was defined as gestational age less than 36 weeks.
\end{tabular}

operation. Five patients did not have reliable postoperative data for calculation of technical performance scores, mainly because they required intraoperative ECMO support and could never have full evaluation of the repair. Four of these 5 were high-risk patients. All 5 patients were excluded from the analyses involving technical performance scores but were included in the analyses involving PRISM III and Aristotle scores and postoperative outcomes.

Six attending surgeons performed 3\%, 10.4\%, 14.1\%, $21.5 \%, 25.2 \%$, and $25.9 \%$ of the operations. A complete postoperative echocardiogram was performed before discharge in all cases. The median age at operation was 5 days (range, 2-42 days). Table 1 summarizes the demographic, morphologic, and procedural characteristics, including preoperative catheter-based interventions (fetal and postnatal). Table 2 summarizes the patients' Aristotle procedure-independent and procedure-dependent factors, including associated procedures performed during the stage I procedure. In-hospital mortality for the entire study group was 19 of $135(14.1 \%)$, or 16 of $132(12.1 \%)$ after exclusion of the 3 patients who initially had biventricular repairs converted to stage I Norwood procedures. Other outcomes, such as use of ECMO, major complications, and hospital and ICU stays, are shown in Table 3 . The technical performance was graded as optimal in 81 cases $(62.3 \%)$, adequate in 26 $(20.0 \%)$, and inadequate in $23(17.7 \%)$. Preoperative and 24- and 48-hour postoperative PRISM III scores ranged from 2 to 24 (median, 8), 8 to 39 (median, 18), and 8 to 31 (median, 17), respectively. Aristotle comprehensive scores ranged from 14.5 to 24.5 (median, 18.5).

\section{Technical Performance and Outcomes}

Patients with inadequate performance had significantly higher mortality, longer hospital and ICU stays, longer ven-
TABLE 2. Aristotle procedure-dependent (most frequent, maximum 5 points), procedure-independent factors, and associated procedures (maximum 5 points)

\begin{tabular}{|c|c|c|}
\hline & No. & $\%$ \\
\hline \multicolumn{3}{|l|}{ Procedure-independent factors } \\
\hline $\begin{array}{l}\text { Preoperative mechanical ventilation } \\
\text { for cardiopulmonary failure }\end{array}$ & 41 & $30.4 \%$ \\
\hline Weight $<2.5 \mathrm{~kg}$ & 21 & $15.6 \%$ \\
\hline Shock resolved at time of surgery & 17 & $12.6 \%$ \\
\hline Extracardiac and genetic anomalies & 11 & $8.1 \%$ \\
\hline Heterotaxy & 5 & $3.7 \%$ \\
\hline Genetic or chromosomal anomaly & 3 & $2.2 \%$ \\
\hline Dextrocardia & 2 & $1.5 \%$ \\
\hline Tracheoesophageal fistula & 1 & $0.7 \%$ \\
\hline Prematurity (32-35 wk) & 8 & $5.9 \%$ \\
\hline Severe prematurity ( $<32 \mathrm{wk})$ & 3 & $2.2 \%$ \\
\hline Renal dysfunction & 5 & $3.7 \%$ \\
\hline \multicolumn{3}{|l|}{ Procedure-dependent factors } \\
\hline Aortic atresia & 53 & $39.3 \%$ \\
\hline Obstructed (intact or restrictive atrial septum) & 23 & $17 \%$ \\
\hline AV valve regurgitation, grade 3 or 4 & 5 & $3.7 \%$ \\
\hline Interrupted aortic arch repair & 3 & $2.2 \%$ \\
\hline TAPVC repair & 3 & $2.2 \%$ \\
\hline $\begin{array}{l}\text { Aberrant right subclavian artery } \\
\text { (except in Sano) }\end{array}$ & 2 & $1.5 \%$ \\
\hline Age $>1$ mo & 2 & $1.5 \%$ \\
\hline \multicolumn{3}{|l|}{ Associated procedures } \\
\hline Aortic valvuloplasty & 15 & $11.1 \%$ \\
\hline Mitral valvuloplasty & 10 & $7.4 \%$ \\
\hline Pulmonary valvuloplasty & 1 & $0.7 \%$ \\
\hline Tricuspid valvuloplasty & 2 & $1.5 \%$ \\
\hline Endofibroelastosis resection* & 15 & $11.1 \%$ \\
\hline
\end{tabular}

$A V$, Atrioventricular; $T A P V C$, total anomalous pulmonary venous connection. *Endofibroelastosis resection was not listed as an associated procedure in the Aristotle system, but it was given the equivalent score for aortic or mitral valvuloplasty; if endofibroelastosis resection was performed with mitral or aortic valvuloplasty, no additional points were given for the endofibroelastosis resection.

tilation time, and greater occurrence of major postoperative complications $(P<.0001$ for each comparison; Table 4$)$. Technical performance also had a significant impact on PRISM III scores at 24 postoperative hours $(P=.003$, median 17 in optimal, 19 in adequate, and 23 in inadequate groups; Figure 2), reflecting a higher degree of physiologic illness severity with inadequate performance. Receiver operating characteristic analysis showed significant ability of technical performance scores to discriminate mortality, with an area under the curve of $0.84(P<.0001 ; 95 \% \mathrm{CI}$, 0.74-0.94).

\section{PRISM III Score and Outcomes}

Preoperative and 24- and 48-hour postoperative PRISM III scores differed significantly between patients who died and those who did not, reflecting a higher degree of illness severity in both the preoperative and immediate postoperative periods among the nonsurvivors (median, 8 vs 12 for 
TABLE 3. Technical performance scores and patient outcomes $(n=130)$

\begin{tabular}{|c|c|c|c|c|}
\hline & Overall & Optimal $(\mathbf{n}=\mathbf{8 1})$ & Adequate $(n=26)$ & Inadequate $(n=23)$ \\
\hline In-hospital mortality (no.) & $19(14.1 \%)$ & $1(1.2 \%)$ & $5(19.2 \%)$ & $8(34.8 \%)$ \\
\hline Hospital stay (d, median and range) & $18(1-293)$ & $15(7-96)$ & $20(4-138)$ & $46(1-293)$ \\
\hline Intensive care stay (d, median and range) & $9(1-293)$ & $8(3-60)$ & $9(3-58)$ & $29(1-293)$ \\
\hline Ventilation time (d, median and range) & $6(1-293)$ & $5(1-47)$ & $7(2-58)$ & $19(1-293)$ \\
\hline Major postoperative complications (no.) & $34 *(25.2 \%)$ & $5(6.2 \%)$ & $9(34.6 \%)$ & $15(65.2 \%)$ \\
\hline Extracorporeal membrane oxygenation (no.) & $25 \dagger(18.7 \%)$ & $3(3.7 \%)$ & $5(19.2 \%)$ & $13(56.5 \%)$ \\
\hline
\end{tabular}

All differences $P<.0001$. *Includes 5 patients without technical performance scores. $\dagger$ Includes 4 patients without technical performance scores.

preoperative PRISM III; $P=.034$; median, 17 vs 25 for $24-$ hour postoperative PRISM III; $P<.0001$; median, 17 vs 21 for 48-hours postoperative PRISM III; $P=.011$ ). Of preoperative and 24- and 48-hour postoperative PRISM III scores, discrimination for mortality was best for the 24-hour postoperative score, with areas under the curve of $0.65(P=.035$; 95\% CI, 0.50-0.81), 0.83 ( $P<.0001 ; 95 \%$ CI, 0.75-0.92), and $0.70(P=.011 ; 95 \% \mathrm{CI}, 0.55-0.84)$, respectively.

\section{Aristotle Comprehensive Score and Outcomes}

Aristotle comprehensive score had a significant association with mortality $(P=.003$, median 18 for live patients vs 21 for dead patients). It also correlated significantly with hospital and ICU stays and with ventilation time

TABLE 4. Factors tested in multivariable analysis for mortality

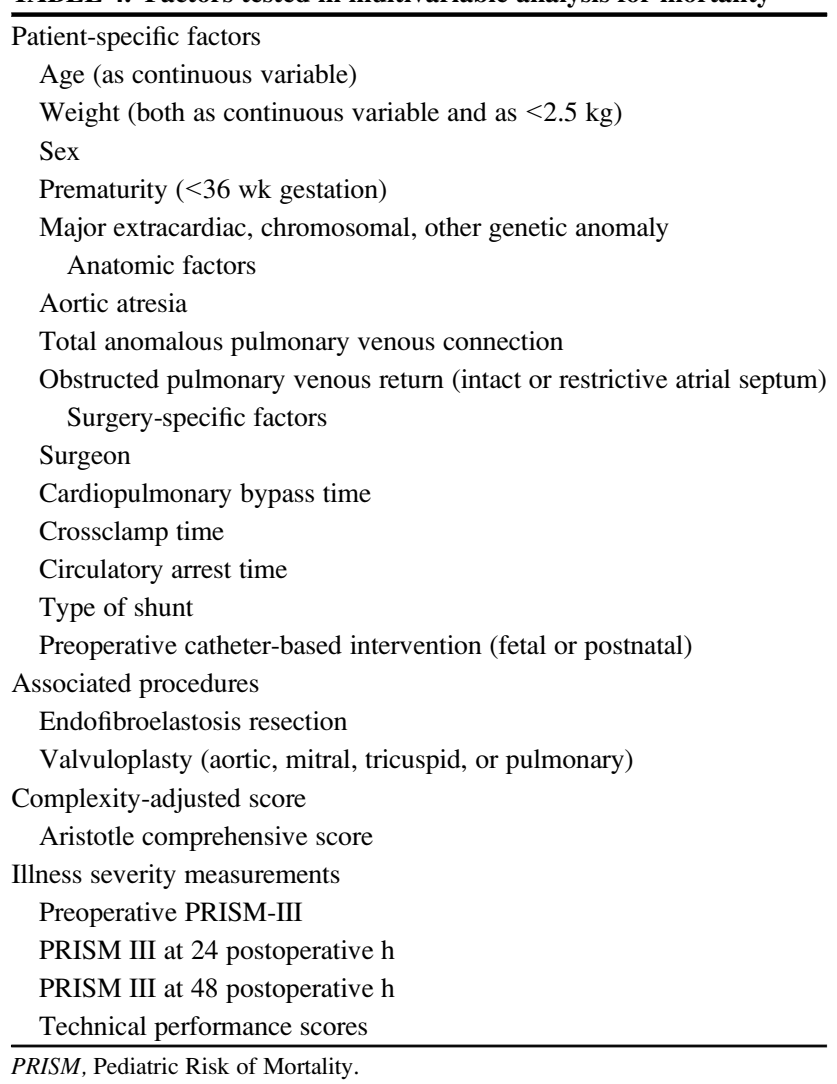

$(P<.0001$ for each correlation test, with Spearman correlation coefficients of $0.330,0.322$, and 0.328 , respectively). Receiver operating characteristic analysis showed significant ability of the Aristotle score in discriminating mortality, with an area under the curve of $0.71(P=.003 ; 95 \% \mathrm{CI}$, 0.59-0.83).

\section{Mortality}

Multivariable analysis. In a univariate analysis, the variables from Table 4 that showed significant correlation with mortality (in addition to PRISM III, Aristotle comprehensive, and technical performance scores) were weight, obstructed pulmonary venous return, and cardiopulmonary bypass time. In the logistic regression models containing 2 explanatory variables simultaneously, the models that included the following performed best according to coefficient of determination $\left(R^{2}\right)$ : technical performance with Aristotle comprehensive score and technical performance with preoperative PRISM III score. For the model including technical performance and Aristotle score, the odds of a patient dying with inadequate and adequate technical performance were 43.8 and 21 times (95\% CI, 5.0-387.1 and 2.3-195.7 times), respectively, the odds of a patient dying with optimal performance. For the model including technical performance and preoperative PRISM III score, the odds of a patient dying with inadequate or adequate technical performance were 42.6 or 20.2 times (95\% CI, 4.8-377.6 and 2.2-187.3 times) the odds of a patient dying with optimal technical performance.

Analyses stratified by technical performance score (Figure 1). A subgroup analysis of the patients according to technical performance scores showed the following: (1) In the optimal technical performance group, there were no differences according to mortality status in preoperative PRISM III score or complexity as measured by Aristotle score (median 8 in live patients vs 14 in dead patients, $P$ $=.198$, and median 17.5 in live patients vs 19.5 in dead patients, $P=.667$, respectively). (2) In the adequate technical performance group, mortality was associated with higher preoperative PRISM III scores and higher Aristotle comprehensive scores (median 6 in live patients vs 16 in dead patients, $P=.006$, and median 17.5 in live patients vs 20.5 in dead patients, $P=.023$, respectively). In the inadequate 


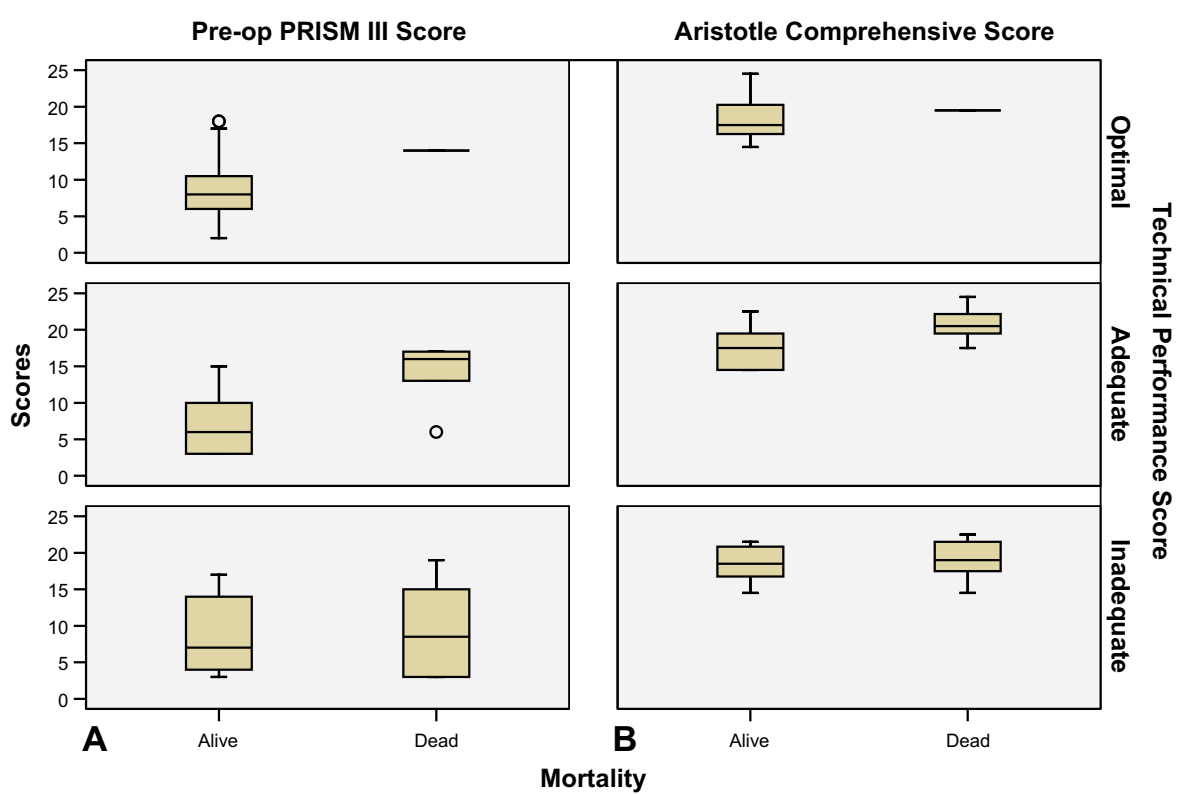

FIGURE 1. Subgroup analysis by technical performance score and preoperative Pediatric Risk of Mortality (pre-op PRISM) III score or Aristotle score versus mortality.

technical performance group, there was no difference in preoperative PRISM III scores or Aristotle scores according to mortality status (median 7 in live patients vs 8.5 in dead patients, $P=.985$, and median 18.5 in live patients vs 19 in dead patients, $P=.648$, respectively).

\section{DISCUSSION}

Measurement of technical competence is inherently difficult. ${ }^{11}$ We previously developed a technical scoring system for common congenital heart procedures ${ }^{12}$ and subsequently

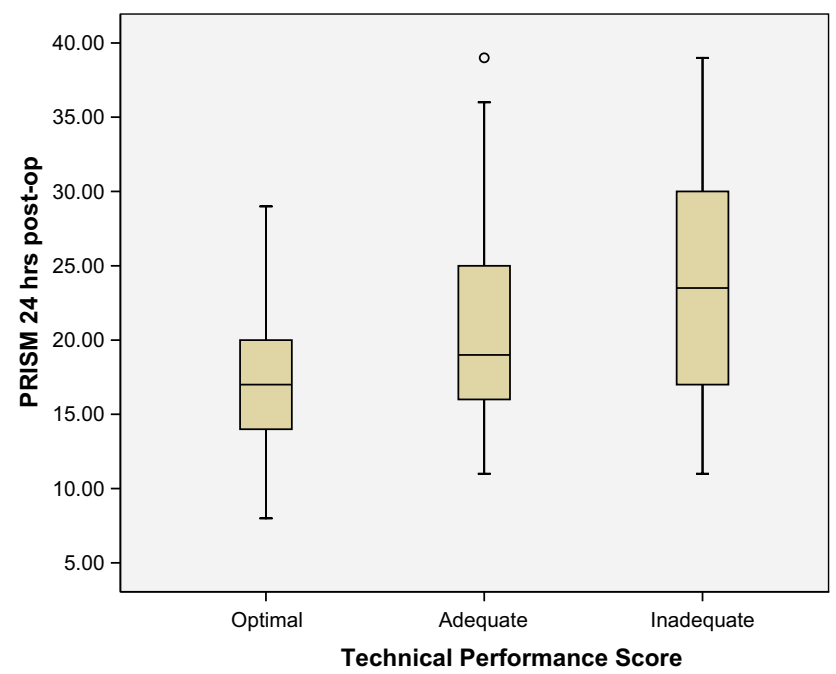

FIGURE 2. Pediatric Risk of Mortality (PRISM) score 24hours postoperative (post-op) and technical performance score. expanded this to the stage I Norwood palliation procedure. ${ }^{1}$ The most recent previous study showed that inadequate technical performance had a significant association with higher mortality and longer hospital stay. This study, which included all patients from 2007, has confirmed those findings and shown a significant association between preoperative physiologic illness severity (measured by PRISM III and partially by Aristotle), case complexity (Aristotle), technical performance, and outcomes. Optimal technical performance attenuated the impact of preoperative illness severity and resulted in good outcomes regardless of preoperative illness severity or case complexity. Inadequate technical performance resulted in poor outcomes regardless of preoperative illness severity or case complexity. An adequate technical performance resulted in good outcomes for lower preoperative illness severity or case complexity and poor outcomes for higher preoperative illness severity or case complexity. In other words, the clinical scenario for patients with adequate technical performance was dictated by baseline physiology and anatomy, whereas outcomes for patients with optimal or inadequate performance were dependent more on the actual technical results, rather than on baseline physiology or anatomy.

A recent review of 412 pancreatic resections showed similar findings. Escalating physiologic risk worsened postoperative morbidity and increased cost, but these effects were attenuated by improved operative performance. ${ }^{13} \mathrm{~A}$ similar interaction, or effect modification, between baseline physiology and technical performance was seen in our study. In our regression model to predict mortality, the best models, according to coefficient of determination $\left(R^{2}\right)$, included both 
technical performance with preoperative PRISM III score and technical performance with Aristotle comprehensive scores. All other risk factors for mortality dropped out.

Interestingly, we found no correlation between preoperative PRISM III or Aristotle comprehensive score and technical performance, suggesting that patient-specific variables such as weight or anatomy are less likely to be responsible for technical performance. As expected, higher postoperative physiologic illness severity scores (PRISM III scores at 24 and 48 hours) were associated with worse technical scores and also with worse outcomes. When compared with the Aristotle score, postop PRISM III scores also performed better in the ability to predict mortality. With the original PRISM scoring system, a previous study had demonstrated this in a prospective fashion. ${ }^{14}$ Technical performance still had the highest predictive value (area under the curve of 0.84), however, illustrating the impact of sound intraoperative techniques. Not surprisingly, inadequate technical performance had a significant correlation with higher PRISM III score at 24 postoperative hours, irrespective of preoperative risk.

There are several important limitations worth mentioning. (1) This was a single-center, retrospective analysis. In addition to the bias inherent in a retrospective study, these results may be institution specific. For example, hospitals with a cardiac surgical team comprising 2 surgeons only or an ICU in which poor results are obtained despite optimal technical performance might not find the same results. (2) The PRISM system was not designed for cyanotic patients and thus has inherent flaws in that respect. ${ }^{15}$ Patients with ECMO support, for example were scored according to their laboratory values, with no additional changes made to the score because of mechanical support. (3) Weight as a continuous variable was significantly associated with mortality in the univariate analysis. Because it is included in the Aristotle score, weight could not be used in the multivariable analysis; however, it is possible that weight alone, a simpler variable, might be a good surrogate for the more complex case complexity calculations. (4) The technical scoring system is an expert opinion-based consensus system that remains somewhat subjective. Some variables that are not entirely under surgeon control had to be included as surrogates for technical performance. For example, it is well documented that the shunt is a significant source of complications in the stage I Norwood procedure; however, objectively judging the technical performance of a shunt is difficult. Downsizing a shunt because of pulmonary overcirculation reduced the score from optimal to adequate, and revision of a shunt because of acute thrombosis resulted in an inadequate score (need for reintervention). Both occurrences clearly affect outcomes negatively, but both could also be the result of patient-specific factors, such as lower then expected pulmonary resistance or a dysfunctional coagulation cascade, both well-documented postoperative occurrences in stage I Norwood procedures but not necessarily the result of poor technical performance. (5) One might argue that because technical performance is the paramount influence on outcomes, preoperative risk optimization by stabilization is not necessary. The concept of preoperative stabilization is prevalent in our ICU, however, and thus all patients were managed according to this concept. Therefore this study could not test that hypothesis. In addition, most surgeons had an even distribution of technical scores (surgeon as risk factor dropped out in the regression model), and thus optimal technical performance is not guaranteed even for the "best" surgeons. (6) These results are specific to the fragile population undergoing the stage I Norwood procedure. Our first study, which looked at technical scores for the arterial switch, ventricular septal defect, tetralogy of Fallot, and complete atrioventricular canal repairs, did not elicit an association between technical scores and clinical outcomes for these procedures, which are also characterized by an immediate postoperative physiologic benefit. ${ }^{12}$ Specifically, the impact of technical performance on mortality may only be valid for the stage I Norwood population.

In conclusion, technical performance emerged as a powerful predictor of clinical outcomes for the stage I Norwood operation. Optimal technical performance significantly attenuated the impact of preoperative illness severity and case complexity, whereas inadequate technical performance resulted in poor outcomes regardless of preoperative status. These findings should be confirmed by other institutions or in a multicenter study.

The following surgeons undertook operations described in this series: Pedro J. del Nido, Emile A. Bacha, Frank A. Pigula, John E. Mayer, Jr, Francis Fynn-Thompson, and Bassem Mora. We thank Kathy Jenkins, MD, and David Clarke, MD, for their support and advice

\section{References}

1. Bacha EA, Larrazabal LA, Pigula FA, Gauvreau K, Jenkins KJ, Colan SD, et al. Measurement of technical performance in surgery for congenital heart disease: the stage I Norwood procedure. J Thorac Cardiovasc Surg. 2008;136:993-7.

2. Stasik CN, Goldberg CS, Bove EL, Devaney EJ, Ohye RG. Current outcomes and risk factors for the Norwood procedure. J Thorac Cardiovasc Surg. 2006;131 412-7.

3. Ashburn DA, McCrindle BW, Tchervenkov CI, Jacobs ML, Lofland GK, Bove EL, et al. Outcomes after the Norwood operation in neonates with critical aortic stenosis or aortic valve atresia. J Thorac Cardiovasc Surg. 2003;125: 1070-82.

4. Forbess JM, Cook N, Roth SJ, Serraf A, Mayer JE, Jonas RA. Ten-year experience with palliative surgery for hypoplastic left heart syndrome. Risk factors related to stage I mortality. Circulation. 1995;92:262-6.

5. Artrip JH, Campbell DN, Ivy DD, Almodovar MC, Chan KC, Mitchell MB, et al. Birth weight and complexity are significant factors for the management of hypoplastic left heart syndrome. Ann Thorac Surg. 2006;82:1252-9.

6. Lacour-Gayet F, Clarke D, Jacobs J, Comas J, Daebritz S, Daenen W, et al. The Aristotle score: a complexity-adjusted method to evaluate surgical results. Eur J Cardiothorac Surg. 2004;25:911-24.

7. Jacobs JP, Lacour-Gayet FG, Jacobs ML, Clarke DR, Tchervenkov CI, Gaynor JW, et al. Initial application in the STS congenital database of complexity adjustment to evaluate surgical case mix and results. Ann Thorac Surg. 2005;79: $1635-49$. 
8. Aristotle Institute [Internet]. Denver: The Institute; c2009. Available at http:// www.aristotleinstitute.org/. Accessed July 10, 2009.

9. Pollack MM, Ruttimann UE, Getson PR. Pediatric risk of mortality (PRISM) score. Crit Care Med. 1988;16:1110-6.

10. Pollack MM, Patel KM, Ruttimann UE. PRISM III: an updated pediatric risk of mortality score. Crit Care Med. 1996;24:743-52.

11. Birkmeyer NJ, Birkmeyer JD. Strategies for improving surgical quality—should payers reward excellence or effort? N Engl J Med. 2006;354(8):864-70.

12. Larrazabal LA, del Nido PJ, Jenkins KJ, Gauvreau K, Lacro S, Colan SD, et al. Measurement of technical performance in congenital heart surgery: a pilot study. Ann Thorac Surg. 2007;83:179-84.
13. Pratt W, Callery MP, Vollmer CM. Optimal surgical performance attenuates physiologic risk in high-acuity operations. J Am Coll Surg. 2008; 207:717-30.

14. Zobel G, Rodl S, Rigler B, Metzler H, Dagar D, Grubbauer H, Beitzke A. Prospective evaluation of clinical scoring systems in infants and children with cardiopulmonary insufficiency after cardiac surgery. J Cardiovasc Surg. 1993;34: 333-7.

15. Mildh L, Pettilä V, Sairanen H, Rautiainen P. Predictive value of paediatric risk of mortality score and risk adjustment for congenital heart surgery score after paediatric open-heart surgery. Interact Cardiovasc Thorac Surg. 2007;6: 628-31. 